\title{
A memristor-based third-order oscillator: beyond oscillation
}

\author{
A. Talukdar $\cdot$ A. G. Radwan $\cdot$ K. N. Salama
}

Received: 1 July 2011/Accepted: 1 August 2011/Published online: 25 August 2011

(c) The Author(s) 2011. This article is published with open access at Springerlink.com

\begin{abstract}
This paper demonstrates the first third-order autonomous linear time variant circuit realization that enhances parametric oscillation through the usage of memristor in conventional oscillators. Although the output has sustained oscillation, the linear features of the conventional oscillators become time dependent. The poles oscillate in nonlinear behavior due to the oscillation of memristor resistance. The mathematical formulas as well as SPICE simulations are introduced for the memristor-based phase shift oscillator showing a great matching.
\end{abstract}

Keywords Memristor $\cdot$ Linear time variant $\cdot$ Oscillator

\section{Introduction}

The three basic circuit elements have recently encountered the missing fourth element- 'memristor', relating between the flux-linkage $(\varphi)$ and the charge $(q)$, when introduced experimentally by HP labs (Strukov et al. 2008) using a thin semiconductor film $\left(\mathrm{TiO}_{2}\right)$ sandwiched between two metal contacts, after the visionary hypothesis given by Chua (1971). According to Strukov et al. (2008) the

A. Talukdar · A. G. Radwan · K. N. Salama $(\bowtie)$ Department of Electrical Engineering, King Abdullah University of Science and Technology, Thuwal, Saudi Arabia e-mail: khaled.salama@kaust.edu.sa

A. Talukdar

e-mail: abdul.talukdar@kaust.edu.sa

A. G. Radwan

e-mail: ahmed.radwan@kaust.edu.sa

A. G. Radwan

Applied Engineering Mathematics,

Cairo University, Giza, Egypt memristive property naturally appears in nanoscale devices and has the potential to replace conventional transistors in memory applications (Snider 2007; Yan et al. 2010; Manem et al. 2010), as well as in cross bar switching applications (Wang et al. 2010; Young et al. 2009).

Parametric oscillator is a well known phenomenon in linear time variant system in control theory which occurs only with externally applied periodical forces as in the case of a child on a swing or quartz oscillator (Komine et al. 2003). Such oscillation does not occur automatically, rather external oscillating inputs are required to pump the system. Previously, the oscillating nature of memristor was analytically modeled under sinusoidal input (Radwan et al. 2010a, b). In our recent work (Talukdar et al. 2010, 2011), the nonlinear dynamics of memristor having oscillating resistance and dynamic poles have been presented for memristor-based Wien oscillator, which requires no external input. This letter tries to generalize those concepts in third-order system with a new insight into realizing parametric oscillation by electrical circuit requiring no input force. Mathematical analyses are carried out showing close agreement with SPICE simulations.

\section{Memristor-based phase shift oscillator}

Conventional resistors are replaced (one at a time) by memristor in the phase shift oscillator (Fig. 1). $R_{\mathrm{M}}$ represents the resistance of memristor. The SPICE model proposed by Biolek et al. (2009) is used to simulate the effect of memristor on phase shift oscillator. The model is implemented as a SPICE subcircuit with the following parameters: $\left(R_{\mathrm{off}}, R_{\mathrm{on}}, D, \mu_{\mathrm{v}}, p\right)=(16 \mathrm{k} \Omega, 0.5 \mathrm{k} \Omega, 10 \mathrm{~nm}$, $\left.10^{-14}, 10\right)$, which are the upper resistance, the lower resistance, the thin film width, the dopant mobility, and the 


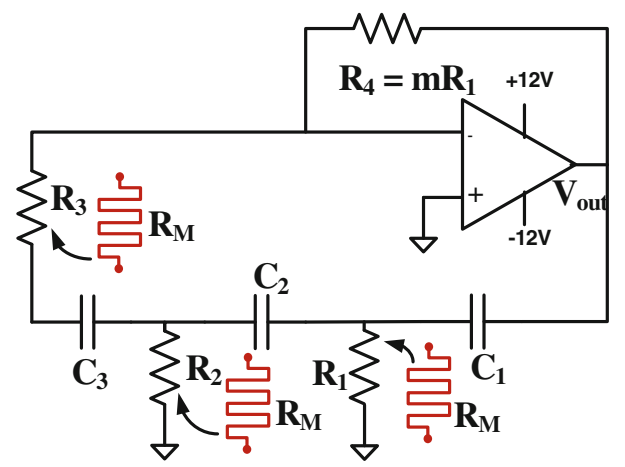

Fig. 1 Schematics of memristor-based phase shift oscillator

nonlinear effect parameter. A parametric study of the initial resistance $R_{\text {init }}$ is introduced to validate the concept for different cases.

\section{Oscillating $\boldsymbol{R}_{\mathbf{M}}$}

From the simulation result, sustained oscillation is found even with oscillating behavior of $R_{\mathrm{M}}$. Though $R_{\mathrm{on}}(0.5 \mathrm{k} \Omega)$ and $R_{\text {off }}(16 \mathrm{k} \Omega)$ are supposed to be the most likely values for $R_{\mathrm{M}}, R_{\mathrm{M}}$ itself shows sustained oscillation with an average value of $R_{\text {avg. }}$. Figure 2 shows both the sustained output oscillation and the oscillating $R_{\mathrm{M}}$ (for $R_{\text {init }}=$ $6.5 \mathrm{k} \Omega$ ) when $R_{1}$ is replaced with $R_{\mathrm{M}}$ keeping $R_{2}$ and $R_{3}$ as $6.5 \mathrm{k} \Omega$. In this figure it is clearly observed that both $R_{2}$ and $R_{3}$ are constant at $6.5 \mathrm{k} \Omega$, whereas $R_{\mathrm{M}}$ oscillates within a definite range from 6.05 to $6.95 \mathrm{k} \Omega$. For this third-orderoscillatory system, $R_{\mathrm{M}}$ can be expressed as:

$R_{\text {max }}-R_{\text {min }} \cong V_{\mathrm{M}} k\left(R_{\text {off }}-R_{\text {on }}\right) /\left(\pi R_{\text {init }} f_{\mathrm{M}}\right)$

where $k$ is given as $k=\left(\mu_{\mathrm{v}} R_{\mathrm{on}}\right) / D^{2} . f_{\mathrm{M}}$ is the frequency of oscillation and $V_{\mathrm{M}}$ is the voltage across $R_{\mathrm{M}} \cdot R_{\max }$ and $R_{\min }$ are the maximum and minimum value of $R_{\mathrm{M}}$. Using (1), the

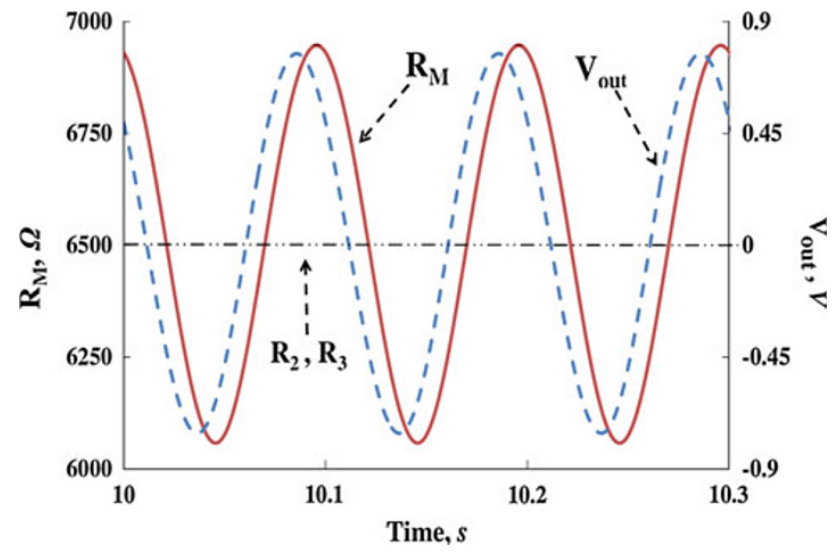

Fig. 2 Simulation results of $V_{\text {out }}, R_{2}, R_{3}$, and $R_{\mathrm{M}}$ for $R_{\text {init }}=6.5 \mathrm{k} \Omega$ when $R_{1}$ is replaced with $R_{\mathrm{M}}$. Straight line, $R_{\mathrm{M}}$; Broken line, $V_{\text {out }}$; dotted and broken line, $R_{2}$ and $R_{3}$

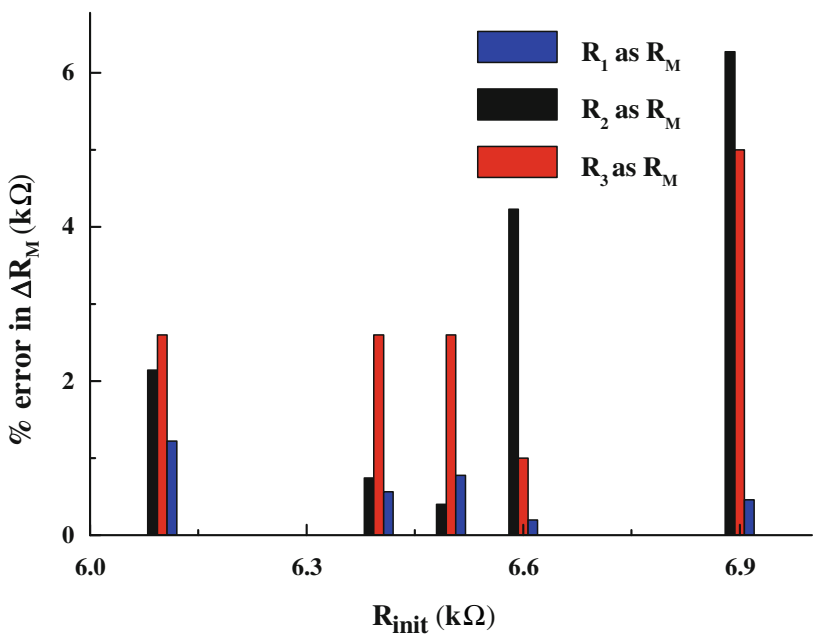

Fig. 3 Percentage error in calculating $\Delta R_{\mathrm{M}}$ for different $R_{\text {init }}$ when one of the resistors is replaced by memristor

amplitude swing of $R_{\mathrm{M}}\left(\Delta R_{\mathrm{M}}=R_{\max }-R_{\min }\right)$ can be estimated for any $R_{\text {init }}$. As $R_{\text {init }}$ and $R_{\text {avg }}$ are almost equal, $\Delta R_{\mathrm{M}}$ will essentially give the values of $R_{\max }$ and $R_{\min }$ as well. When $R_{2}$ or $R_{3}$ is replaced with $R_{\mathrm{M}}$, similar oscillation is found but with different amplitude swing. In case of $R_{2}, R_{\mathrm{M}}$ oscillates from $6.28 \mathrm{k} \Omega$ to $6.55 \mathrm{k} \Omega$ (when $R_{\text {init }}=6.5 \mathrm{k} \Omega$ ). For $R_{3}$, the amplitude swing of $R_{\mathrm{M}}$ is observed from $6.37 \mathrm{k} \Omega$ to $6.47 \mathrm{k} \Omega$. Figure 3 compares the percentage error in calculating $\Delta R_{\mathrm{M}}$ using (1) for different $R_{\text {init }}$. The maximum error in estimating $R_{\mathrm{M}}$ from (1) is found as $1.22 \%$ (for $R_{1}$ as $R_{\mathrm{M}}$ ), for $R_{2}$ as $R_{\mathrm{M}}$ the error is $6.3 \%$, and for $R_{3}$ as $R_{\mathrm{M}}$ the maximum error is $5 \%$. This oscillating $R_{\mathrm{M}}$ can be the realization of periodically changing parameter. Usually in parametric oscillators, the parameters are externally applied periodically to initiate oscillation but in this memristor-based phase shift oscillator, no external means are required to have the parameter $\left(R_{\mathrm{M}}\right)$ oscillating with time.

\section{Oscillating poles}

The characteristic equation of this system can be expressed as:

$a s^{3}+b s^{2}+c s+d=0$

$a=R_{\mathrm{M}} R^{2} C^{3}(1+m), \quad d=1$

$b=4 R_{\mathrm{M}} R C^{2}+2 R^{2} C^{2}, \quad c=2 R_{\mathrm{M}} C+3 R C$,

where $m=R_{4} / R_{1}$ (Fig. 1). There will be three roots which will be defined by the coefficients $a, b, c$, and $d$. Two of the roots are complex conjugate and the other one is real. These roots/poles determine the stability of the system, where conventionally it is believed that all the poles will be fixed in the left half of the $s$ plane. But surprisingly in this system poles are found oscillating. From (3), it is obvious that $a, b$, and $c$ are 


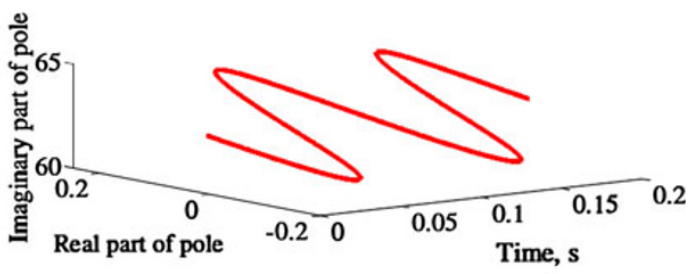

(a)

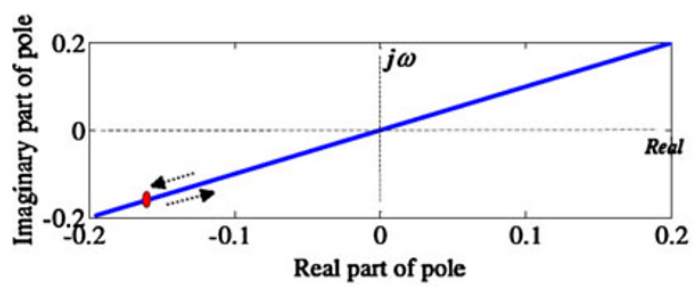

(b)

Fig. 4 a Oscillating pole with time. b $s$ plane representation of oscillating pole

dependent on $R_{\mathrm{M}}$ and as $R_{\mathrm{M}}$ is sinusoidal oscillating, the poles are expected to be dynamic. In Fig. $4 \mathrm{a}$ (when $R_{1}$ is replaced by $R_{\mathrm{M}}$ ), the oscillating behavior of one of the conjugate poles is plotted. Here both the real and imaginary part of complex poles are oscillating with time which means in the $s$ plane that pole will not be fixed, rather it will oscillate from the left half $s$ plane to the right half $s$ plane as shown in Fig. $4 \mathrm{~b}$ (red circle symbolizes pole). When the pole shifts to the right half plane then the oscillating $R_{\mathrm{M}}$ fetches the pole from the unstable region to the stable region of the left half plane, but then $R_{\mathrm{M}}$ changes its value which tends to pull back the poles towards the right half plane and this periodic incident continues. These oscillations in all three poles are observed for every $R_{\text {init }}$ as well as when $R_{2}$ and $R_{3}$ are also replaced by $R_{\mathrm{M}}$.

\section{Frequency of oscillation, $f_{\mathrm{M}}$}

Due to the sinusoidally oscillating $R_{\mathrm{M}}, f_{\mathrm{M}}$ can be modeled as:

$f_{\mathrm{M}}=\frac{1}{2 \pi C \sqrt{R\left(R+5\left(R_{\mathrm{avg}} \pm \Delta R_{\mathrm{M}}\right)\right)}}$

observing (4), $f_{\mathrm{M}}$ will have a range of values from maximum, $f_{\mathrm{M}, \text { max }}$ (when $R_{\mathrm{M}}=R_{\min }$ ) to minimum, $f_{\mathrm{M} \text {,min }}$ (when $\left.R_{\mathrm{M}}=R_{\max }\right) . f_{\mathrm{M}}$ will have a range of frequencies for oscillation but the output oscillation set itself to that fre-

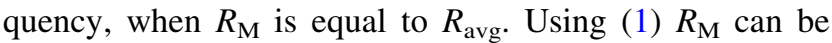
approximated to be used in (4) to calculate $f_{\mathrm{M}}$ for corresponding $R_{\text {init }}$. The maximum percentage of error in estimating $f_{\mathrm{M}}$ is $0.4 \%$ when $R_{1}$ is replaced by $R_{\mathrm{M}}$. In case of $R_{2}$ and $R_{3}$ replaced by memristor, the maximum error in calculating $f_{\mathrm{M}}$ is 0.45 and $0.1 \%$, correspondingly.

\section{Conclusion}

In spite of having oscillating resistance of memristor, and dynamic behavior of poles, sustained oscillation is observed in this memristor-based third-orderoscillatory system. For the first time, these unprecedented behaviors of memristor in oscillation can become the circuit implementation of parametric oscillation without any external means. Memristor can be considered as a better candidate than resistor for low-frequency oscillator which is widely used in programmable frequency-based counter, random number generator, synthesizing bass, random number generator, and sophisticated portable system.

Open Access This article is distributed under the terms of the Creative Commons Attribution License which permits any use, distribution and reproduction in any medium, provided the original author(s) and source are credited.

\section{References}

Biolek Z, Boilek D, Biolkova V (2009) SPICE model of memristor with nonlinear dopant drift. Radioengineering 18(2):210

Chua LO (1971) Memristor-the missing circuit element. IEEE Trans Circuit Theory CT-18(5):507

Komine V, Galliou S, Makarov A (2003) A parametric quartz crystal oscillator. IEEE Trans Ultrason Ferroelectr Freq Control 50(12): $1656-1661$

Manem H, Rose GS, He X, Wang W (2010) Design considerations for variation tolerant multilevel CMOS/Nano memristor memory. In: Proceedings of the ACM Great Lakes symposium on VLSI, May 2010, Providence, pp 287-292

Radwan AG, Zidan MA, Salama KN (2010) On the mathematical modeling of memristors. In: International conference on microelectronics (ICM), pp 284-287

Radwan AG, Zidan MA, Salama KN (2010) HP memristor mathematical model for periodic signals and DC. In: IEEE International Midwest Symposium on Circuits and Systems, Seattle, USA, pp 861-864

Snider G (2007) Architecture and methods for computing with reconfigurable resistor crossbars (Patent style), US Patent 7203789

Strukov DB, Snider GS, Stuwart DR, Williams RS (2008) The missing memristor found. Nature 453:80-83

Talukdar A, Radwan AG, Salama KN (2010) Time domain oscillating poles: stability redefined in memristor-based wien-oscillators. In: International Conference on Microelectronics (ICM), pp 288-291

Talukdar A, Radwan AG, Salama KN (2011) Generalized model for memristor-based wien family oscillators. Microelectron $\mathrm{J}$ 42:1032-1038

Wang FZ, Helian N, Wu S, Lim M-G, Guo Y, Parker MA (2010) Delayed switching in memristors and memristive systems. Electron Device Lett IEEE 31(7):755-757

Yan ZB, Li SZ, Wang KF, Liu J-M (2010) Unipolar resistive switching effect in $\mathrm{YMn} 1-\delta \mathrm{O}_{3}$ thin films. Appl Phys Lett 96(1):012103-012103-3

Young JH, Lee JY, Choi SY, Kim JW (2009) Microscopic origin of bipolar resistive switching of nanoscale titanium oxide thin films. Appl Phys Lett 95(16):162108-162108-3 